\title{
Modeling of Surge in Free-Spool Centrifugal Compressors: Experimental Validation
}

\author{
Jan Tommy Gravdahl* \\ Norwegian University of Science and Technology, N-7491 Trondheim, Norway \\ Frank Willems ${ }^{\dagger}$ \\ TNO Automotive, 2600 JA Delft, The Netherlands \\ Bram de Jager \\ Eindhoven University of Technology, 5600 MB Eindhoven, The Netherlands \\ and \\ Olav Egeland ${ }^{\S}$ \\ Norwegian University of Science and Technology, N-7491 Trondheim, Norway
}

\begin{abstract}
The derivation of a compressor characteristic, and the experimental validation of a dynamic model for a variable speed centrifugal compressor using this characteristic, are presented. The dynamic compressor model of Fink et al. is used, and a variable speed compressor characteristic is derived by the use of energy transfer and loss analysis. It is demonstrated that taking into account the losses due to friction, incidence, mixing, and blade loading results in compressor characteristics that closely match the measured characteristics. The simulated response of the dynamic model was found to be in excellent agreement with the experimental results, both for set point changes using fuel flow and blow off and for surge oscillations. Analysis of the power spectrum of the in-surge rotational speed and pressure oscillations reveal that the simulated nonlinear oscillations match experimental values up to the third harmonic, both with respect to frequency and amplitude.
\end{abstract}

\section{Introduction}

$\mathbf{C}$ OMPRESSORS are widely used for the pressurization of fluids. Applications include air compression in gas turbines used in power plants and aircraft and marine propulsion. Another important application is pressurization and transportation of gas in pipelines and in the process and chemical industries. If a compressor is operated at a mass flow below a certain lower limit called the surge line, the compressor may go into an unstable operational mode called surge. Surge is characterized by a limit-cycle oscillation in compressor flow and pressure rise. This oscillation is also present in the rotational speed. Operation during surge often results in considerably reduced efficiency and performance and can lead to high blade and casing stress levels. Surge can also induce vibrations in other components of the compression system such as, for example, the connected piping. In summary, surge oscillations are, at the very least, unwanted, and can, in extreme cases, even damage the compressor. The standard industrial solution to this problem is to use control systems that prevent the operating point from entering the unstable regime of the compressor map. Such schemes are called surge avoidance schemes, and can be implemented, for example, by the use of recirculation or blowoff valves. However, surge can be actively controlled, that is, the unstable equilibria below the surge line can be stabilized by the use of feedback from one or several sensors via a control law to one or more actuators. The first report of such a scheme can be found in Ref. 1. Recent overviews of work on compressor instabilities and surge control can be found in Refs. 2, 3, and 4.

In the field of active compressor control, there is a need for models that can be employed in controller design. A classical result in the

\footnotetext{
Received 1 July 2002; revision received 2 February 2004; accepted for publication 25 March 2004. Copyright (C) 2004 by the American Institute of Aeronautics and Astronautics, Inc. All rights reserved. Copies of this paper may be made for personal or internal use, on condition that the copier pay the $\$ 10.00$ per-copy fee to the Copyright Clearance Center, Inc., 222 Rosewood Drive, Danvers, MA 01923; include the code 0748-4658/04 \$10.00 in correspondence with the CCC.

*Associate Professor, Department of Engineering Cybernetics.

${ }^{\dagger}$ Research Scientist, Powertrains Department, P.O. Box 6033. 513 .

Associate Professor, Department of Mechanical Engineering, P.O. Box

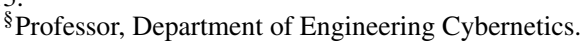

field of compressor surge modeling is the axial compressor model of Greitzer, ${ }^{5}$ where a dynamic model for mass flow and pressure rise was presented. In Ref. 6, it was shown that the Greitzer model is also applicable to centrifugal compressors. Because compressors are variable-speed machines, and surge is commonly encountered during speed transients and set point changes, it is of interest to investigate the influence of speed transients on the surge dynamics and on the compressor dynamics in general. A model describing this interaction was developed by Fink et al. in Ref. 7, where rotational speed was included as a state in the model. Based on the work of Fink et al., a similar model was derived in Ref. 8, using a compressor characteristic derived by the use of energy transfer and loss analysis.

This paper presents an experimental study validating the dynamic model from Ref. 8, which was based on balance laws, flow dynamics, and thermodynamics, and included loss terms due to incidence loss and friction. Instead of using a measured characteristic, we propose to derive the characteristic mathematically. The motivation behind this approach is to identify the structure of the governing equations, with a main focus on the compressor characteristic, to exploit that structure and the properties of the nonlinearities, that is, the compressor characteristic, in the design of active surge controllers. This is an approach to controller design that has been taken in a number of other fields. For an overview, consult Ref. 9. A recent result in the compressor control field can be found in Ref. 10. In this context, it is of interest to study what physical effects shape the compressor characteristic, especially the destabilizing effect of the incidence loss. If the model is to be useful in controller design, however, it is necessary to validate the model experimentally. Initial experiments confirming this model were presented in Ref. 11, wherein this model is further developed. In this work, additional loss terms, as well as an improved incidence loss model, are included to achieve a close fit between the model data and the experimental data, both for the compressor characteristic and for transients.

\section{Experimental Setup}

\section{A. Compression System}

To study the dynamic behavior of turbomachinery, a laboratoryscale gas-turbine installation was erected in the Energy Technology Laboratory of Eindhoven University of Technology. This 
installation, which is documented in Ref. 12, is built around a BBC VTR 160L turbocharger. It consists of a single-stage, centrifugal compressor with a vaned diffuser and exit scroll that is mounted on the same rotational axis as the single-stage, axial expander. When the expander is mounted on the same axis as the compressor, it can deliver the power to pressurize the incoming gas. To increase the net mechanical power provided at the shaft, the temperature of the working gas is raised by burning fuel in the combustion chamber.

For studies involving compressor surge, the gas-turbine installation is mostly used in the configuration shown in Fig. 1, with the dashed connecting line removed. Similar to that in Ref. 7, the mass flows through the compressor, and the compressor and expander are now decoupled; the compressor pressurizes the incoming air, which is discharged via the compressor blowoff valve into the atmosphere. The volume of the piping between the compressor exit and the blowoff valve now acts as the plenum volume. This modified configuration offers the following benefits. First, the occurring surge oscillations are assumed to be less harmful for the equipment because the compressor discharges in a relatively small plenum volume compared to the large vessel of the standard gas-turbine installation. Second, Ref. 7 showed that, in this small-volume case, the steady-state compressor characteristics can be determined up to smaller mass flows. Third, if the compressor and expander mass flows are coupled, the number of steady operating points reduces significantly because a power and mass balance holds between both components. ${ }^{12,13}$ In the studied system, externally supplied compressed air flows via the expander throttle valve into the combustion chamber where natural gas is added and burned. Because the compressor and expander mass flows can be varied independently, the modified system can, in principle, be operated at any desired operating point. However, in this configuration, the rotational speed can be varied up to $25,000 \mathrm{rpm}$ due to the limited mass flow rate of the externally supplied compressed air.

For higher compressor speeds, the gas pressurized by the compressor can supplement the externally supplied compressed air by the use of the dashed connecting line and by closing the compressor blowoff valve. Because this configuration includes the large vessel, surge is much more powerful and is of lower frequency. No extended surge measurements are possible, otherwise damage to the machine could easily occur. Some characteristic turbocharger data are listed in Table 1. Note that angles are measured from the tangent, and that the blade inlet angle $\beta_{1 b}$ is the angle at the rms inlet diameter $D_{1}=\sqrt{ }\left(D_{h 1}^{2}+D_{t 1}^{2}\right) . V_{p}$ is the volume of the piping between the compressor outlet and the blowoff valve. In this paper, the compressor is operated at speeds varying between $N=16,000$ and 22,000 rpm. Greitzers $B$ parameter,

$$
B=\left(U_{2} / 2 a\right) \sqrt{V_{p} / A_{1} L_{c}}
$$

where $U_{2}$ is the impeller tip speed and $a$ is the sonic velocity at ambient conditions, is then found to vary between $B=0.58$ and 0.81 .

\section{B. Measurement System}

To determine the steady-state performance of the compressor, the compression system is equipped with several temperature probes and pressure transducers, as shown in Fig. 1. Moreover, steady expander and compressor blowoff mass flows can be determined from two instrumented orifices, whereas the rotational speed of the impeller is measured with a semiconductor pulse tachometer. The angular displacements of the stems of several valves are also registered. The signals $Y_{b}$ and $Y_{g}$ are measures for the opening area of the blowoff valve and fuel valve, respectively. Because these valves have a linear flow characteristic, the mass flow through the valve is proportional to the valve position. ${ }^{12}$ For data acquisition, a National

Table 1 Parameters of the VTR 160L

\begin{tabular}{|c|c|c|}
\hline Parameter & Value & $\overline{\text { Unit }}$ \\
\hline \multicolumn{3}{|c|}{ Impeller } \\
\hline No. of blades $Z$ & 20 & . \\
\hline Slip factor $\sigma$ & 0.9 & \\
\hline Inlet diameter tip $D_{t 1}$ & 0.106 & $\mathrm{~m}$ \\
\hline Inlet diameter hub $D_{h 1}$ & 0.054 & $\mathrm{~m}$ \\
\hline Exit diameter $D_{2}$ & 0.180 & $\mathrm{~m}$ \\
\hline Exit width $b_{2}$ & 0.007 & $\mathrm{~m}$ \\
\hline Blade inlet angle $\beta_{1 b}$ & 40 & $\operatorname{deg}$ \\
\hline Blade exit angle $\beta_{2 b}$ & 90 & deg \\
\hline \multicolumn{3}{|c|}{ Diffuser } \\
\hline No. of vanes $z$ & 45 & \\
\hline Inlet diameter $D_{3}$ & 0.215 & $\mathrm{~m}$ \\
\hline Outlet diameter $D_{4}$ & 0.258 & $\mathrm{~m}$ \\
\hline Vane inlet angle $\alpha_{2 b}$ & 28 & deg \\
\hline Vane outlet angle $\alpha_{3}$ & 40 & deg \\
\hline \multicolumn{3}{|c|}{ System } \\
\hline Plenum volume $V_{p}$ & 0.0203 & $\mathrm{~m}^{3}$ \\
\hline Duct area $A_{1}$ & 0.00956 & $\mathrm{~m}^{2}$ \\
\hline Duct length $L_{c}$ & 2.45 & $\mathrm{~m}$ \\
\hline
\end{tabular}

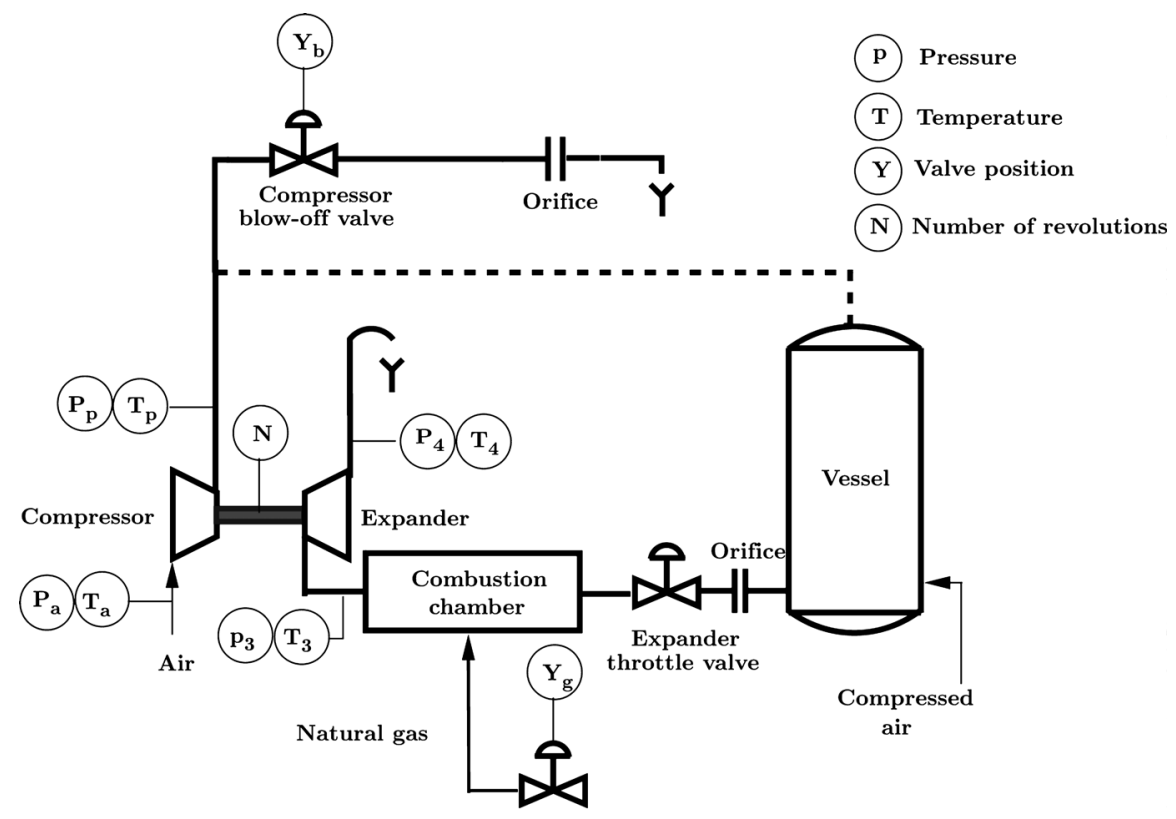

Fig. 1 Scheme of experimental setup. 
Instruments board is plugged in a measurement personal computer. LabVIEW software controls the data storage on hard disk and realtime process monitoring on the computer screen. This system runs at 200-Hz sampling frequency. A second personal computer, equipped with a dSPACE DS1103 control board is used to run (surge) controllers and to gather a subset of the available data at high speeds, because it is set at a $1-\mathrm{kHz}$ sampling frequency. Results of the use of active surge control by the use of blowoff valves on this compressor rig can be found in Ref. 14. Compressor transients are observed via measurements of the high-frequency response pressure probe at the compressor outlet, the compressor blowoff valve's rotation angle sensor, and the rotational speed transducer. Reliable, transient mass flow measurements are not available. The accuracies of the sensors used in this paper are as follows: pressure transducers, $0.5 \%$; valve positions, $1.0 \%$; rotational speed, $0.5 \%$; and turbine temperatures, $1.0 \%$.

\section{Model}

The model of Fink et al. ${ }^{7}$ can be written as

$$
\begin{gathered}
\dot{p}_{p}=\left(a_{01}^{2} / V_{p}\right)\left(\dot{m}-\dot{m}_{t}\right), \quad \ddot{m}=\left(A_{1} / L_{c}\right)\left(p_{02}-p_{p}\right) \\
\dot{\omega}=(1 / J)\left(\tau_{t}-\tau_{c}\right)
\end{gathered}
$$

where $p_{p}$ is the plenum pressure, $\dot{m}$ is the compressor mass flow, $\omega$ is the angular velocity of the compressor shaft, $p_{02}$ is the pressure downstream of the compressor, $a_{01}$ is the inlet stagnation sonic velocity, $L_{c}$ is the length of compressor and duct, $A_{1}$ is the area of the impeller eye (used as reference area), $V_{p}$ is the plenum volume, $J$ is the central moment of inertia of the shaft including mounted parts, $\dot{m}_{t}$ is the throttle mass flow, $\tau_{t}$ is the turbine drive torque, and $\tau_{c}$ is the compressor torque. The first two Eqs. (1) are equivalent to the model in Ref. 5. The model is derived by calculation of the mass balance of the plenum volume, integration of the one dimensional Euler equation (the momentum balance) over the length of the compressor and ducts, and calculation of the torque balance of the rotating shaft. The velocity triangle at the inducer, or the impeller eye, is shown in Fig. 2, and the velocity triangle at the impeller tip is shown in Fig. 3. The velocities and angles in the derivation that follows can be seen in Figs. 2 and 3.

\section{A. Compressor and Turbine Torques}

For turbomachines, applied torque equals the change in angular momentum of the fluid:

$$
\tau_{c}=\dot{m}\left(r_{2} C_{\theta 2}-r_{1} C_{\theta 1}\right)
$$

where $r_{1}=D_{1} / 2, r_{2}=D_{2} / 2$, and $C_{\theta 1}$ and $C_{\theta 2}$ are the tangential components of the gas velocities $C_{1}$ and $C_{2}$. By the use of Eq. (2), the power delivered to the fluid can be calculated as

$$
\dot{W}_{c}=\omega \tau_{c}=\dot{m} \Delta h_{02}
$$

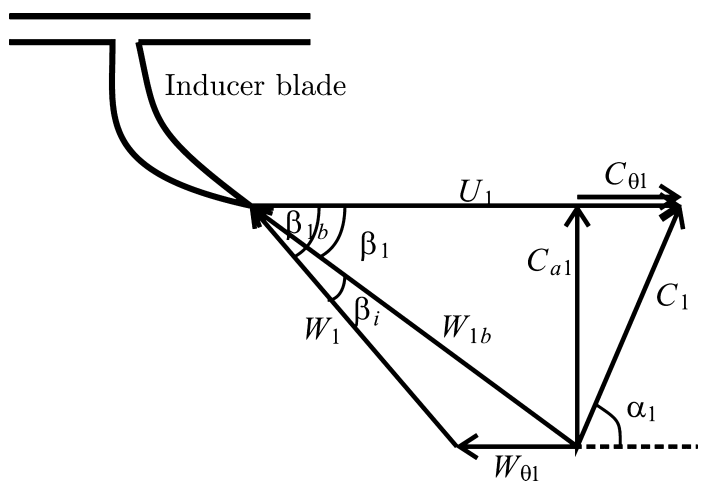

Fig. 2 Velocity triangle at the inducer, section through the inducer at radius.

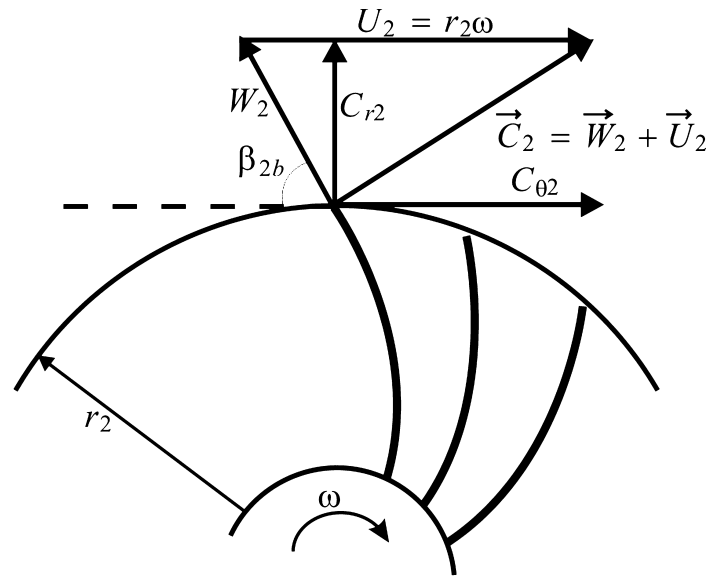

Fig. 3 Velocity triangle at the impeller tip.

where

$$
\Delta h_{02}=\left(U_{2} C_{\theta 2}-U_{1} C_{\theta 1}\right)
$$

is the specific enthalpy delivered to the fluid without taking account of losses, and $U_{i}=r_{i} \omega, i=1,2$, is the tangential speed of the impeller at diameter $D_{i}$. In this paper, a radially vaned impeller is considered with $\beta_{2 b}=90 \mathrm{deg}$, and there is no prewhirl, that is, $\alpha_{1}=90 \mathrm{deg} \Rightarrow C_{\theta 1}=0$. These assumptions are valid for the compressor of the VTR 160. However, the model can easily be extended to handle both backsweep and prewhirl. It now follows that

$$
\Delta h_{02}=\sigma U_{2}^{2}
$$

where

$$
\sigma=C_{\theta 2} / U_{2}
$$

is the slip factor. The compressor torque is then given by

$$
\tau_{c}^{+}=\dot{m} r_{2} C_{\theta 2}=\dot{m} r_{2} \sigma U_{2}, \quad \dot{m}>0
$$

The torque calculated in Eq. (5) is for forward flow. However, the compressor may enter deep surge, and there is need for an expression for the compressor torque at negative mass flow. It will be assumed that the compressor torque of a centrifugal compressor in reversed flow can be calculated by the use of Euler's turbine equation:

$$
\tau_{c}^{-}=\dot{m}\left(r_{1} C_{\theta 1}-r_{2} C_{\theta 2}\right)=-\dot{m} r_{2} \sigma U_{2}, \quad \dot{m}<0
$$

Combination of Eqs. (5) and (6) gives

$$
\tau_{c}=|\dot{m}| r_{2} \sigma U_{2}
$$

Note that the torque calculated in Eq. (7) is an idealization. When the flow approaches zero, recirculation will occur inside the impeller, increasing the work input requirement and, therefore, the torque. In reversed flow, $r_{1} C_{\theta 1}$ will initially be large, and, as flow increases, $r_{1} C_{\theta 1}$ will reduce to zero. These effects are not captured by the current model. Also, external losses such as windage and disk friction will prevent the torque from dropping to zero. These torques can be included as additional load torques in Eq. (1), but they are assumed not to have a dominant effect here. The torque characteristic of the VTR 160 compressor, calculated with Eq. (7), is shown in Fig. 4.

In Ref. 7, a constant turbine torque was used for the study of the transients of the compressor system. Because the turbine torque is dependent on the time-varying system states $\dot{m}$ and $U_{2}$, this approach will not be used here. Instead, the turbine drive torque is found by division of turbine power by angular velocity:

$$
\tau_{t}=\frac{P_{t}}{\omega}=\frac{\eta_{m} \dot{m}_{\text {turbine }} c_{p, t} \Delta T_{0, \text { turbine }}}{2 \pi N}
$$

where $\Delta T_{0, \text { turbine }}=T_{t, \text { in }}-T_{t, \text { out }}$ is the stagnation temperature drop across the turbine; $\eta_{m}$ is the turbine mechanical efficiency, which is usuall very high $\left(\eta_{m}=0.99\right.$ begin typical $) ; \dot{m}_{\text {turbines }}$ is the turbine mass flow; $c_{p, t}$ is the turbine specific heat under constant pressure; and $N$ is the rotational speed of the shaft. 


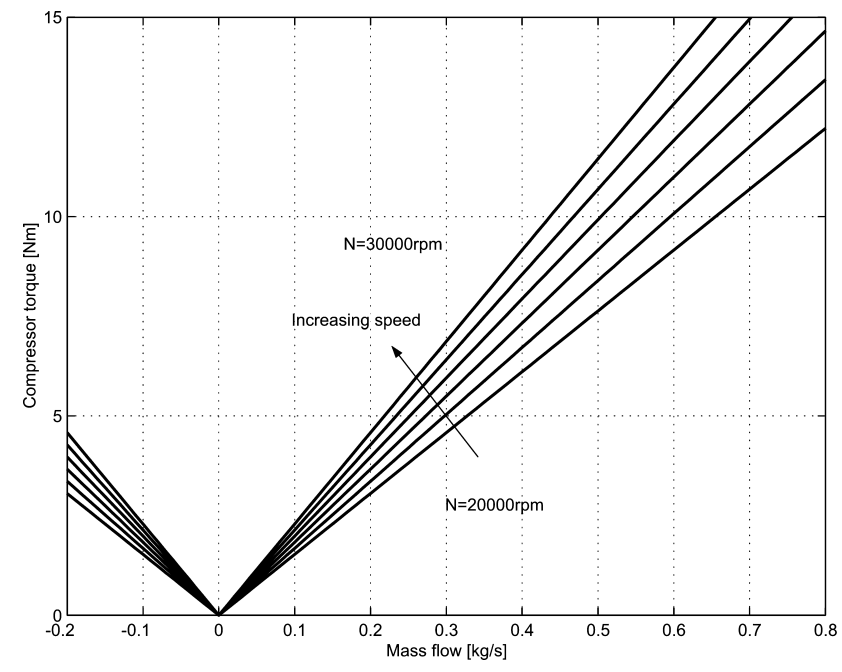

Fig. 4 Idealized compressor torque.

\section{B. Energy Losses in the Compressor}

Notice that due to $\beta_{2 b}=90 \mathrm{deg}$, the ideal specific enthalpy $\Delta h_{02}$ in Eq. (4) is independent of mass flow $\dot{m}$ and is a function of speed only. Ideally, we would have the same specific enthalpy rise for all mass flows. However, due to various losses, the enthalpy rise is not constant, and we will now include this in the analysis.

According to Ref. 15, the two major losses, expressed as specific enthalpies, are 1) incidence losses, $\Delta h_{i}$ and 2) fluid friction losses, $\Delta h_{f}$. These losses are introduced into the compressor characteristic in the model (1) because they play important roles of determining the stability of operating points. The incidence loss destabilizes because it gives rise to the positive slope of the compressor characteristic, and the friction loss stabilizes in the sense that a higher friction loss will shift the surge line to the left, although at the cost of lower pressure rise. For details on this, consult either Ref. 16 or Ref. 15. Thus, the incidence losses and fluid friction losses play an important role in shaping the compressor characteristic and determining the region of stable operation for the compressor, and we proceed to derive detailed models for these losses. In Ref. 11, a preliminary validation of both model and experiment was presented, and it was concluded that the loss model should be refined to get a better match. With this in mind, we take the blade loading loss $\Delta h_{\text {bld }}$ and the mixing loss $\Delta h_{\text {mix }}$ into account. Also, the model of the incidence loss is modified to take into account the increased loss at high incidence angles.

\section{Incidence Loss}

The loss due to off-design incidence angle plays an important role in shaping the compressor characteristic. To calculate this loss, we will, due to its simplicity, use the method of Ref. 17. The method was originally developed for prediction of the off-design performance of radial turbines and was adapted to compressors in Ref. 18 and later in Ref. 15. The velocity of the incoming gas relative to the inducer is denoted $W_{1}$. In off-design operation, there will be a mismatch between the fixed blade angle $\beta_{1 b}$ and the direction of the flow $\beta_{1}=\beta_{1}\left(U_{1}, C_{1}\right)$. The angle of incidence is defined by $\beta_{i}=\beta_{1 b}-\beta_{1}$. As the gas enters the inducer, its velocity instantaneously changes its direction to comply with the constant blade inlet angle $\beta_{1 b}$. The direction is changed from $\beta_{1}$ to $\beta_{1 b}$, and the incidence loss is calculated as the kinetic energy $W_{\theta 1}^{2} / 2$ associated with the tangential component $W_{\theta 1}$ of the velocity. Because of continuity, the radial component of the velocity must remain unchanged. Many authors, see, for example, Ref. 16, report that the incidence loss increases faster than to the power of two when the flow is significantly above or below design flow. A positive constant $\delta<1$ is now introduced to take this into account. The incidence loss is now calculated as

$$
\Delta h_{i i}=\frac{1}{2}\left(U_{1}-\frac{\cot \beta_{1 b} \dot{m}}{\rho_{01} A_{1}}\right)^{2+\delta} u^{-\delta}
$$

where $u$ is the unit velocity. A detailed derivation of the case $\delta=0$ may be found in Ref. 8 . The losses in the vaned diffuser can be modeled in a similar manner, by the use of a velocity triangle for the diffuser inlet. It is assumed that the velocity of the fluid entering the diffuser is instantaneously changed to comply with the fixed diffuser inlet angle $\alpha_{2 b}$. The direction is changed from $\alpha_{2}$ to $\alpha_{2 b}$, and the kinetic energy $C_{3 i}^{2} / 2$ associated with the tangential component $C_{3 i}$ of the velocity is lost. That is, the diffuser incidence loss can be expressed as

$$
\Delta h_{i d}=\frac{1}{2}\left(\sigma U_{2}-\cot \alpha_{2 b} C_{a 3}\right)^{2+\delta} u^{-\delta}
$$

The velocity $C_{a 3}$ is calculated under, the assumption of incompressible flow in the vaneless space.

\section{Friction Loss}

According to Ref. 16, loss due to friction in the impeller can be calculated as

$$
\Delta h_{f i}=f_{i}\left(l_{i} / D_{i}\right) /\left(\bar{W}_{i}^{2} / 2\right)
$$

where $\bar{W}_{i}$ is the mean value of the flow velocity in the impeller, $f_{i}$ is the impeller friction factor, $l_{i}$ is the mean impeller channel length, and $D_{i}$ is the impeller mean hydraulic channel diameter. For calculation of the friction factor, we use Haalands explicit formula (see Ref. 19):

$$
1 / f_{i}^{\frac{1}{2}}=-1.8 \log \left\{6.9 / \operatorname{Re}+\left[\left(\epsilon / D_{i}\right) / 3.7\right]^{1.1}\right\}
$$

The Reynolds number used is $R e=U_{2} b_{2} / v$, where $b_{2}$ is the impeller tip width and $v$ is the kinematic viscosity at the impeller inlet. This was recommended in Ref. 20 as being a representative value of the Reynolds number for a centrifugal compressor stage. The mean hydraulic channel diameter $D_{i}$ is defined as $D_{i}=4 A_{i} / a_{i}$, where the cross section area $A_{i}$ and wetted perimeter $a_{i}$ are mean values for the passage. For the impeller, the mean hydraulic diameter is set to the average of the inducer inlet hydraulic diameter and the impeller exit hydraulic diameter. The friction loss $\Delta h_{f d}$ in the diffuser is calculated accordingly, such that

$$
\Delta h_{f d}=f_{d}\left(l_{d} / D_{d}\right)\left(\bar{W}_{d}^{2} / 2\right)
$$

where $\bar{W}_{d}$ is the mean value of the flow velocity in the diffuser, $f_{d}$ is the diffuser friction factor, $l_{d}$ is the mean diffuser channel length, and $D_{d}$ is the diffuser mean hydraulic channel diameter.

\section{Mixing Loss}

The theory of the jet/wake model and details on the mixing loss can be found in Ref. 21. According to Ref. 22, the mixing loss of Ref. 21 can be calculated as

$$
\Delta h_{\text {mix }}=\frac{1}{1+\tan ^{2} \beta_{2}}\left(\frac{1-\varepsilon_{\text {wake }}-b^{*}}{1-\varepsilon_{\text {wake }}}\right)^{2} \frac{C_{2}^{2}}{2}
$$

where $\beta_{2}$ is the impeller exit flow angle given by

$$
\beta_{2}=\tan ^{-1}\left(C_{r 2} / \sigma U_{2}\right)
$$

$\varepsilon_{\text {wake }}$ is the fraction of the blade to blade space occupied by the wake (in the present model, set to a value of 0.25 ), $b^{*}$ is the ratio of the diffuser inlet width and the impeller exit width (in the present model, set to a value of 1 ). 
4. Blade Loading Loss

According to Ref. 23, the blade loading loss can be calculated as

$$
\Delta h_{\mathrm{bld}}=0.05 D_{f}^{2} U_{2}^{2}
$$

where

$$
D_{f}=1-\frac{W_{2}}{W_{1 t}}+\left(3 \Delta h_{02}\right) / 4 U_{2}^{2}\left(\frac{W_{1 t}}{W_{2}}\right)\left[\frac{Z}{\pi}\left(1-\frac{D_{1 t}}{D_{2}}\right) 2 \frac{D_{1 t}}{D_{2}}\right]
$$

is the diffusion factor according to Ref. 22 , and $Z$ is the number of impeller blades.

\section{Computation of the Compressor Characteristic}

The pressure rise in the compressor increases from the stagnation pressure $p_{01}$ at the inlet to the stagnation pressure $p_{02}$ at the outlet. Generally, this rise in stagnation pressure can be obtained in an isentropic process $1 \rightarrow 2 s$ that involves an increase in the stagnation enthalpy $\Delta h_{02 s}=h_{02 s}-h_{01}$. The pressure increase in the compressor is not isentropic, and there is an increase in entropy due to losses. To account for this, the compression from $p_{01}$ to $p_{02 s}$ is modeled as an isentropic process in series with an adiabatic process $2 s \rightarrow 2$ at constant enthalpy that accounts for the drop in stagnation pressure. This results in the following description: First, there is an isentropic process $1 \rightarrow 2 s$ that ends in a state with pressure $p_{02 s}$ and stagnation enthalpy $h_{02 s}$. Then, there is an adiabatic process, that accounts for the entropy increase and ends in a state with pressure $p_{02}$.

The total process $1 \rightarrow 2$ is illustrated in Fig. 5, and

$$
\begin{aligned}
\Delta h_{02 s}= & \Delta h_{02}+\Delta h_{\text {loss }} \\
= & \Delta h_{02}+\Delta h_{i i}+\Delta h_{f i}+\Delta h_{i d}+\Delta h_{f d}+\Delta h_{\text {mix }} \\
& +\Delta h_{\text {bld }}=\sigma U_{2}^{2}
\end{aligned}
$$

Here $\Delta h_{i i}$ and $\Delta h_{i d}$ are the incidence losses defined in Eqs. (9) and (10), $\Delta h_{f i}$ and $\Delta h_{f d}$ are the friction losses defined in Eqs. (11) and (13), $\Delta h_{\text {mix }}$ is the mixing loss from Eq. (14), $\Delta h_{\text {bld }}$ is the blade loading loss from Eq. (15), and $\Delta h_{02 s}$ is the change in specific stagnation enthalpy that contributes to the acceleration of the gas in the duct. Actually, the various enthalpy terms in Eq. (17) are functions of the operating point, but for reasons of notational simplicity, the arguments $\dot{m}$ and $\omega$ of the terms $\Delta h(\dot{m}, \omega)$ are dropped in the following. The stagnation pressure ratio is derived from the stagnation temperature ratio of the isentropic process according to

$$
p_{02} / p_{01}=\left(T_{02} / T_{01}\right)^{\kappa /(\kappa-1)}
$$

An expression for $T_{02} / T_{01}$ is found from

$$
\begin{array}{r}
T_{02} / T_{01}=h_{02} / h_{01}=\left(h_{01}+\Delta h_{02}\right) / h_{01} \\
=1+\Delta h_{02} / h_{01}=1+\Delta h_{02} / c_{p} T_{01}
\end{array}
$$

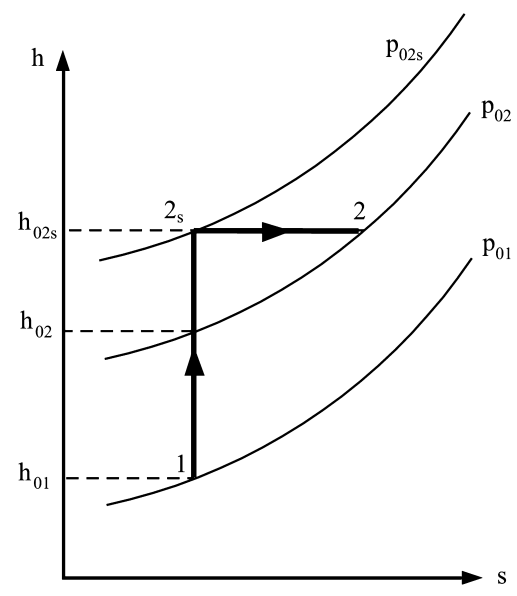

which gives

$$
\Psi_{c}(\dot{m}, w)=\frac{p_{02}}{p_{01}}=\left[1+\frac{\Delta h_{02}(\dot{m}, w)}{c_{p} T_{01}}\right]^{\kappa /(\kappa-1)}, \quad \dot{m} \geq 0
$$

where $\Delta h_{02}(\dot{m}, \omega)$ is calculated from Eq. (17).

The compressor characteristic (20) is valid for positive flow, but during deep surge cycles flow reversal occurs, and so we need an expression for the compressor characteristic in this case. Following Ref. 24, we regard the compressor in reversed flow as a throttling device with a positive pressure bias and model it as

$$
\Psi_{c}(\dot{m}, \omega)=c_{n} \dot{m}^{2}+\psi_{c 0}(\omega), \quad \dot{m}<0
$$

where a suitable value for $c_{n}$ is to be selected. The bias $\psi_{c 0}(\omega)$ is calculated by insertion of $\dot{m}=0$ in Eq. (20). The mass flow $\dot{m}_{t}$ through the throttle is modeled as

$$
\dot{m}_{t}=k_{t} \sqrt{p_{p}-p_{01}}
$$

where $k_{t}$ is the throttle gain proportional to throttle opening. The model

$$
\begin{gathered}
\dot{p}_{p}=\left(a_{01}^{2} / V_{p}\right)\left(\dot{m}-\dot{m}_{t}\right) \\
\ddot{m}=\left(A_{1} / L_{c}\right)\left[\Psi_{c}(\dot{m}, w) p_{01}-p_{p}\right] \\
\dot{w}=(1 / J)\left(\tau_{t}-\tau_{c}\right)
\end{gathered}
$$

given in Eq. (1) can now be completed by the use of Eqs. (7), (6), (8), (20), (21), and (22) for the terms $\tau_{c}, \tau_{t}, \Psi_{c}(\dot{m}, \omega)$, and $\dot{m}_{t}$. The compressor duct area $A_{1}$ and the plenum volume $V_{p}$ are determined from compressor rig geometry. The compressor duct length was chosen such that the surge frequency of the simulations matched the measured frequency. A similar approach was used in Ref. 25, which employed an effective duct length to match the surge frequency in an axial compression rig, and by Ref. 14 for the same rig as in this paper. In the present paper, a value of $L_{c}=2.45 \mathrm{~m}$ was found, whereas Ref. 14 used $L_{c}=1.8 \mathrm{~m}$ for the same rig. It is known that the surge frequency depends on the shape of the characteristic. The difference in the duct lengths is due to the use of different approximations of the compressor characteristics in the present work and Ref. 14.

\section{Comparison with Experimental Results}

The response of the model (23) was compared to the measured response from the laboratory compression system in three different experiments. First, the calculated compressor characteristic in Eq. (20) was compared to the measured characteristic for three different rotational speeds. Then set-point changes were done with both the blowoff valve and the fuel supply valve, and the results were compared to simulations. Finally, the compressor was driven into surge, and the measured surge oscillation was compared to a corresponding simulation.

\section{A. Compressor Characteristic}

In these experiments, the compressor characteristic was measured for three speed lines $(N=18,000 ; 21,000$; and 23,000 rpm. For each speed, the measurements started at high mass flow, and the flow was gradually reduced until surge was encountered. The measurements are shown with $x$ interconnected with thin, solid lines in Fig. 6. A calculated compressor map is shown with thick, solid lines in Fig. 6. These speed lines were calculated with Eq. (20). For both the impeller and diffuser incidence loss, the parameter $\delta$ in Eq. (9) and (10) was set to $\delta=0.04$. For negative flows, the compressor characteristic was calculated with Eq. (21) with $c_{n}$ chosen as $c_{n}=10$. The branch of the compressor characteristic where the flow is negative was not measured, and the choice of $c_{n}$ is made to have approximately the same pressure rise at $\dot{m}= \pm 0.1 \mathrm{~kg} / \mathrm{s}$. There is some uncertainty in this choice of $c_{n}$, but a $50 \%$ variation in $c_{n}$ was found to have minimal influence on the results. The dashed line represents the line between the maxima of the calculated speed lines, which is assumed to be the surge line. Depending on the ratio of the slopes 
of the compressor characteristic and the throttle characteristic, the compressor can be operated in a small range to the left of the maximum, however, according to Ref. 26, the peak of the compressor characteristic provides a convenient working approximation for the surge point. As can be seen from Fig. 6, calculation and measurement agree very well, except at the far right of each speed line. Also, due to the refined calculations for the incidence loss and the inclusion of the mixing loss and the blade loading loss, the agreement between calculation and experiments in the present paper is much better than that in Ref. 11. Because the speed lines were measured by lowering the mass flow until surge occurred, is seen that the surge line is predicted quite well by the calculated speed lines.

\section{B. Set-Point Changes}

Two different types of set-point changes were performed, first with the blowoff valve and then with the fuel valve. The transient measurements were then compared to simulations. Because of difficulties in measuring the turbine exit temperature $T_{t, \text { out }}$, the temperature drop over the turbine $\Delta T_{0, \text { turbine }}$ was calculated from the compressor torque $\tau_{c}$ at constant rotational speed.

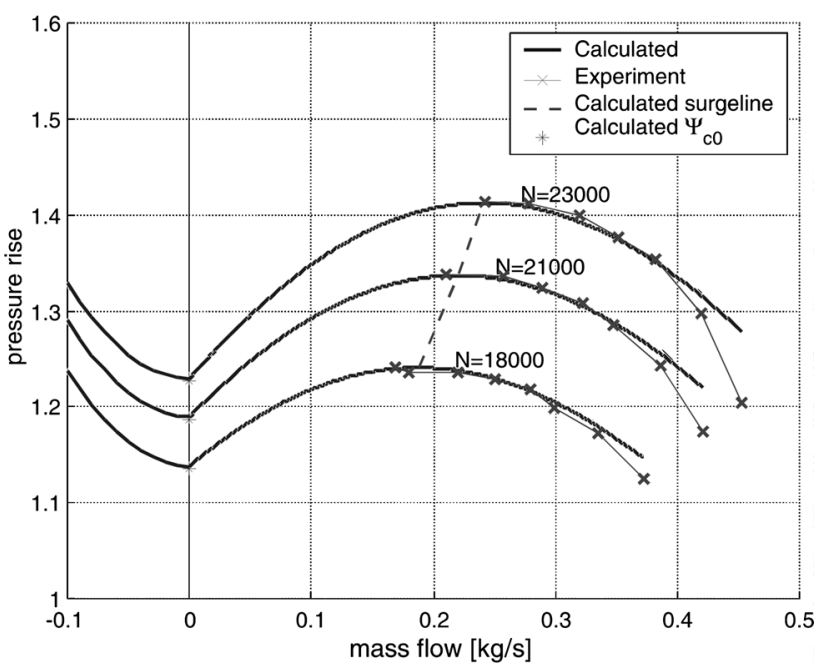

Fig. 6 Compressor characteristics.

\section{Blowoff Valve}

In this experiment the blowoff valve was used to change the set point. The measurements and corresponding simulations are shown in Fig. 7. At $t=5.7 \mathrm{~s}$, the blowoff valve position was changed from $Y_{b}=3.485 \mathrm{~V}$ to $Y_{b}=4.395 \mathrm{~V}$, and back again at $t=52 \mathrm{~s}$. From the mass flow measurements it was found that this corresponds to a step in $k_{t}$ from 0.0015 to 0.0022 . The blowoff valve is modeled as a step and a rate limiter. The valve is rather slow, with a maximum slew rate of $0.11 \mathrm{~V} / \mathrm{s}$. As can be seen from Fig. 7, the simulation and measurement are in good agreement, except for the steady state of the rotational speed. This is most likely due to the difficulties in calculation of the drive torque from measured turbine temperature drop, or due to the assumptions made during calculation of the compressor torque. The spikes seen in the speed measurements in Fig. 7, and also Fig. 8, are due to a grounding problem in the buttons commanding the opening of the fuel supply valve and the blowoff valve.

\section{Fuel Supply Valve/Drive Torque}

In this experiment, the fuel supply valve was used to change the set point. This is equivalent to manipulation of the drive torque. The measurements and corresponding simulations are shown in Fig. 8. At $t=4.1 \mathrm{~s}$, the fuel supply valve position was changed from $Y_{g}=2.06 \mathrm{~V}$ to $Y_{g}=1.68 \mathrm{~V}$, and to $Y_{g}=2.134 \mathrm{~V}$ at $t=48.6 \mathrm{~s}$. This corresponds to a step in turbine temperature drop $\Delta T_{0 \text {,turbine }}$ of $-3.06 \mathrm{~K}$ (from $\Delta T_{0, \text { turbine }}=35.53 \mathrm{~K}$ to $\Delta T_{0, \text { turbine }}=32.47 \mathrm{~K}$ ) at $t=4.1 \mathrm{~s}$ and $3.30 \mathrm{~K}$ (from $\Delta T_{0, \text { turbine }}=32.47 \mathrm{~K}$ to $\Delta T_{0, \text { turbine }}=$ $35.77 \mathrm{~K})$ at $t=48.6 \mathrm{~s}$. The temperature drops were computed from the calculated compressor torque. From the mass flow measurements, it was found that $k_{t}=0.0015$. The turbine responded relatively slowly to a change in fuel supply, and the unmodeled build up of turbine torque was modeled by a first-order dynamic model with a time constant of $8 \mathrm{~s}$ between the fuel valve position and the drive torque $\tau_{d}$. This time constant was found by when the step response was studied. As shown in Fig. 8, the measurement and simulation for this kind of set-point change were in good agreement.

\section{Surge Experiment}

In the surge experiment, the compressor was initially operating close to the surge line, and was then driven into surge closing the blowoff valve. During the surge cycles, pressure and rotational speed were logged by the use of the dSPACE DS1103 control board.
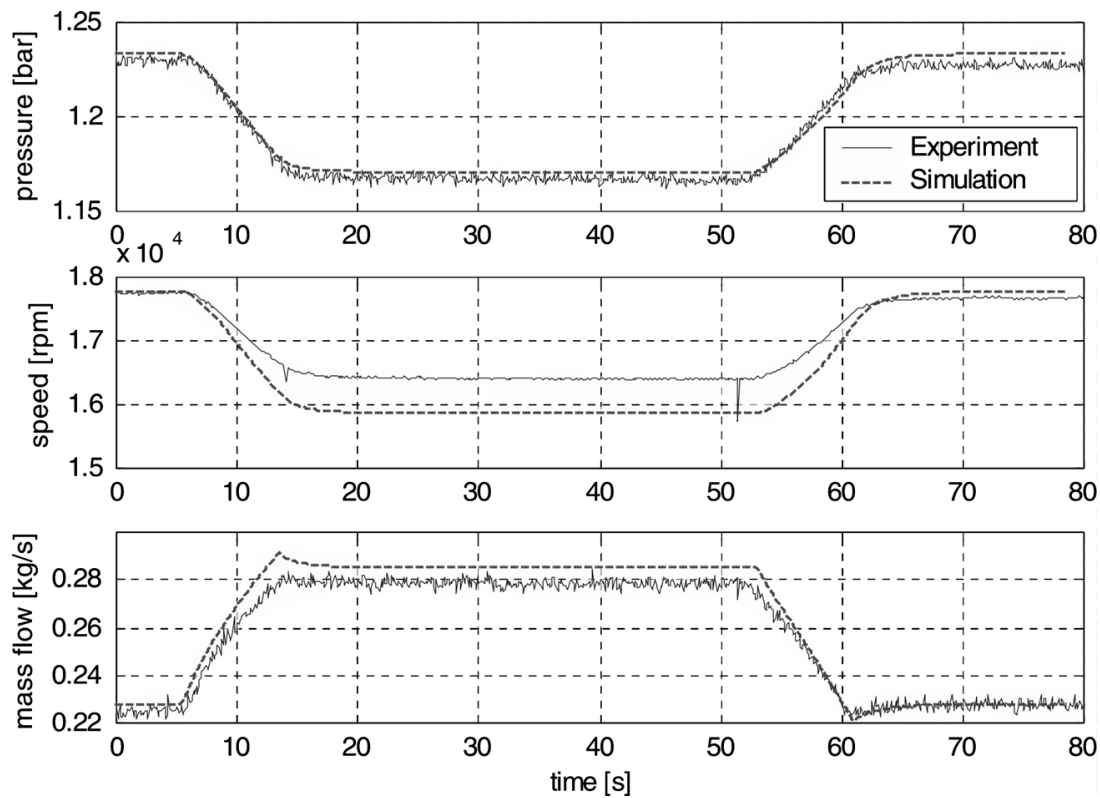

Fig. 7 Changing the set point using the blowoff valve. 

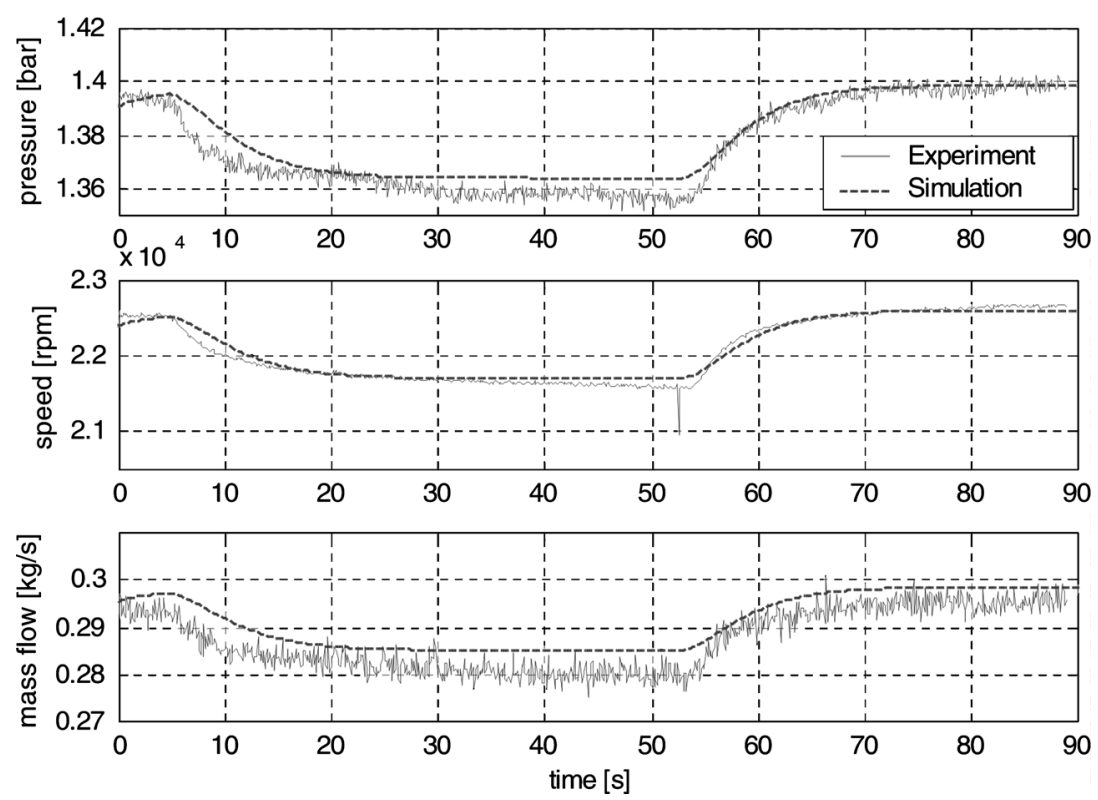

Fig. 8 Changing the set point using the fuel valve.
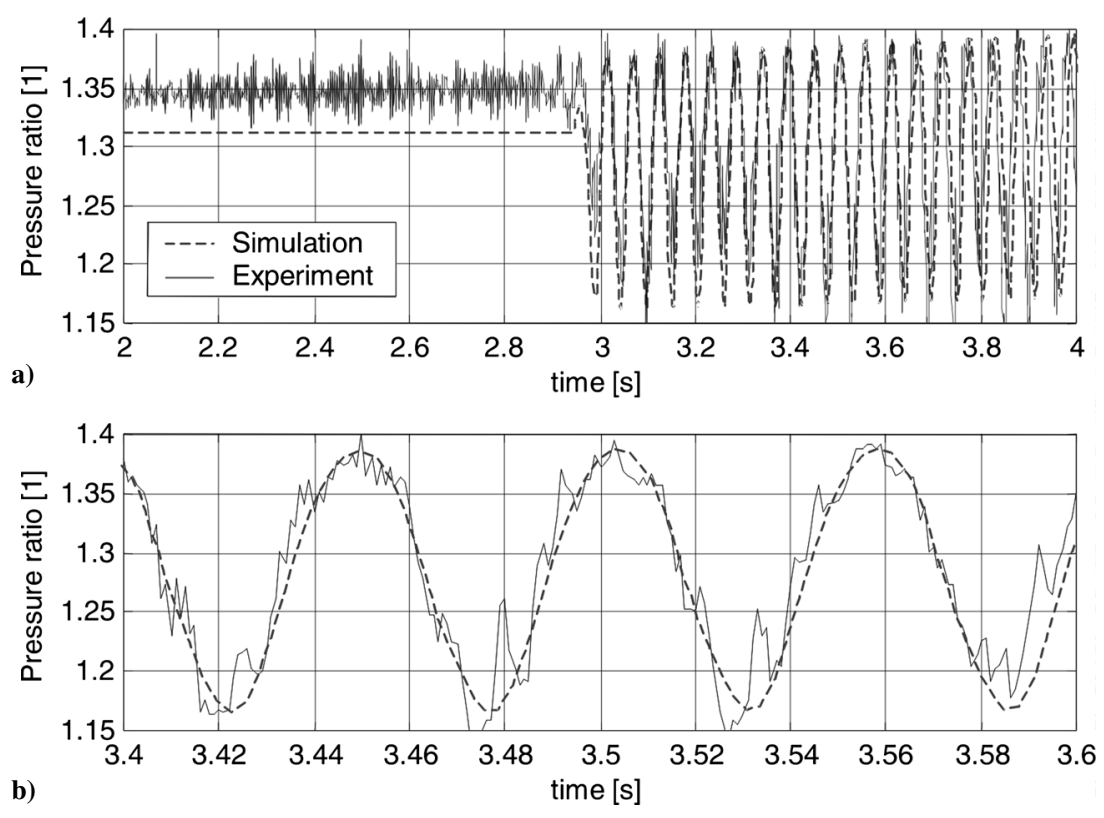

Fig. 9 Measured — and simulated, - ..- pressure oscillations during surge.

\section{Transients}

In Fig. 9a the pressure transient when the compressor enters surge is shown and compared to a simulation of the same situation (dashed lines). Because of the uncertain location of the surge line, the exact start of the surge oscillation is not precisely captured by the model. The timescale of the simulated pressure oscillations has been shifted by $\Delta t=0.25 \mathrm{~s}$. A more detailed comparison is shown in Fig. $9 \mathrm{~b}$, which is a magnified version of Fig. 9a. When the compressor was operating in surge, the rotational velocity of the shaft was also oscillating. The amplitude of this oscillation depended on the size of the plenum volume $V_{p}$. Because we operated with a relatively small volume, it was to be expected that these oscillations were of a low amplitude. Because of the coupling between the states in the model (23), it is expected that the rotational speed of the shaft will oscillate when the compressor is surging. Simulations show that operating the compressor in surge at a speed of $N=22,000 \mathrm{rpm}$, the amplitude of the surge induced speed variations was $40 \mathrm{rpm}$, as shown in Fig. 10. Note from the upper plot in Fig. 10, the measured oscillations in rotational speed were so small that they were not distinguishable from the noise in the measured signal. The increase in speed when the compressor entered surge was because the load torque of the compressor decreases with decreasing mass flow.
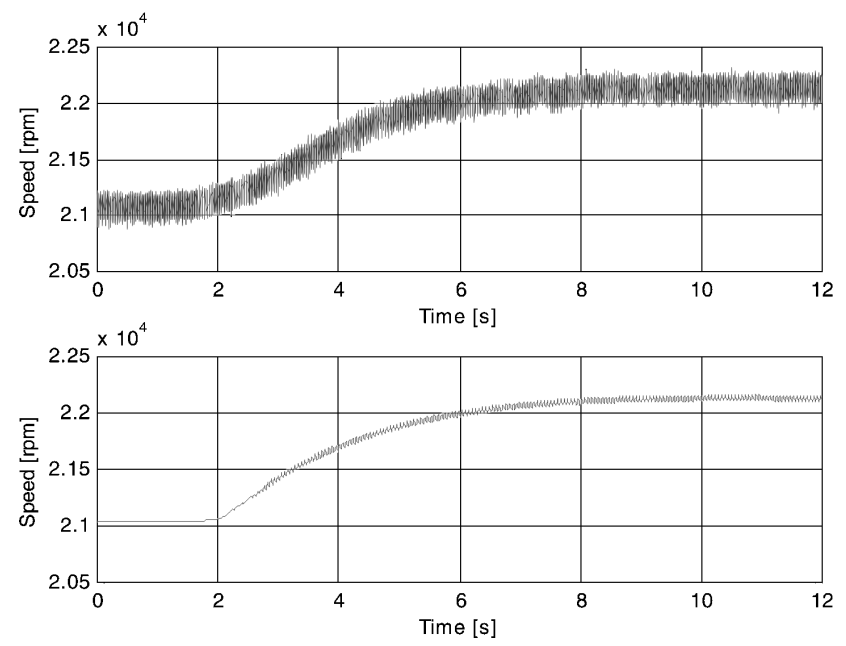

Fig. 10 The a) measured and b) simulated rotational speed when the compressor was driven into surge. 


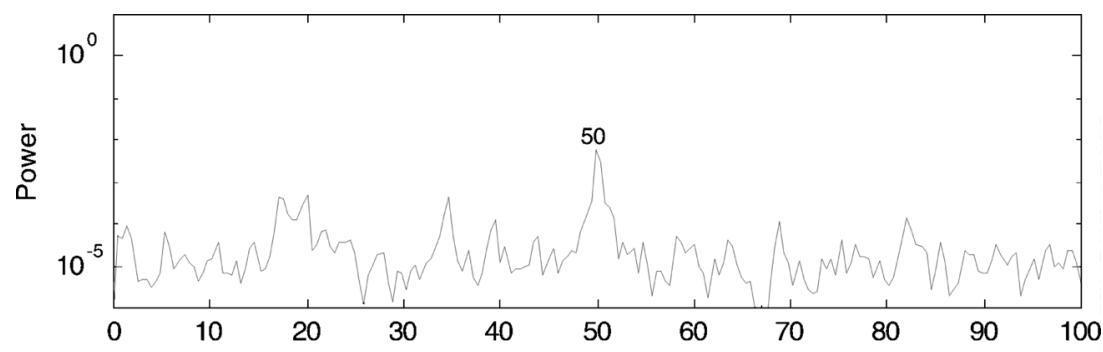

a)

Frequency $[\mathrm{Hz}]$

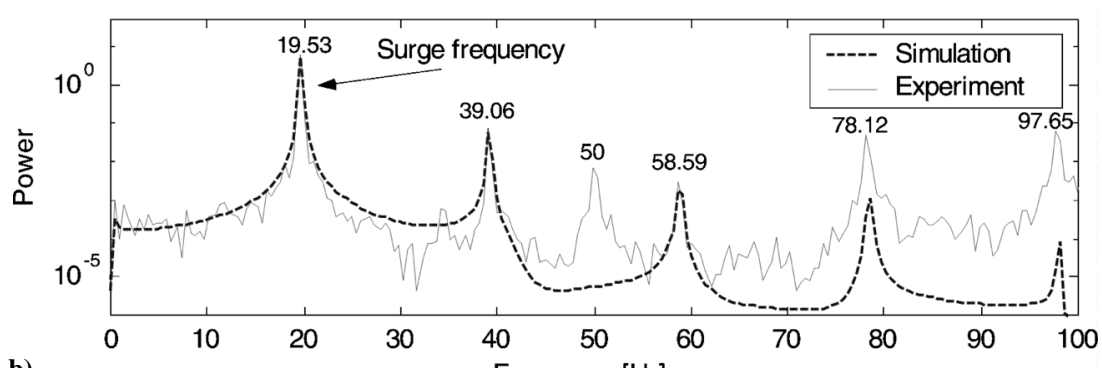

b)

Frequency $[\mathrm{Hz}]$

Fig. 11 Measured and simulated surge pressure signal: a) noise level before surge and b) power spectrum.
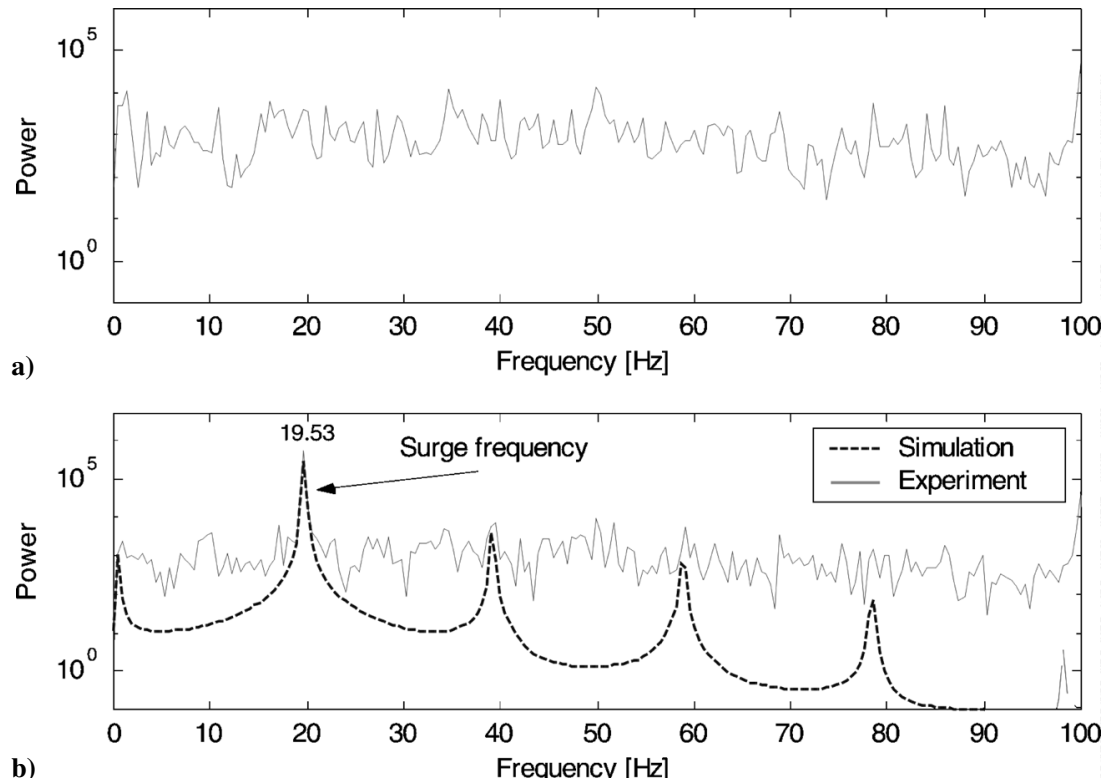

Fig. 12 Measured and simulated surge speed signal: a) noise level before surge and b) power spectrum.

\section{Power Spectra}

To investigate further the validity of the simulations, the power spectra of the pressure and speed signals were calculated. This was done with the spectrum routine in MATLAB ${ }^{\circledR}$. The measurements in the surge experiments were sampled at $1 \mathrm{kHz}$, and the corresponding simulations were computed with a fixed time step of $10^{-3} \mathrm{~s}$. The time series were divided into three overlapping subseries, and the discrete fourier transform were calculated via a $\beta$-valued Kaiser window (see Ref. 27) with $\beta=1$ and a specified sampling rate of $1 \mathrm{kHz}$. The choice of window was made because of its narrow main lobe width and low relative side lobe level. To facilitate precise location of the peaks in the power spectra, a DFT length of 2048 was used to get good frequency resolution. The power spectrum of the plenum pressure is shown in Fig. 11. In the upper part the spectrum of the pressure signal before surge is shown. The power spectrum of the in-surge pressure signal is shown in of Fig. $11 \mathrm{~b}$, where the frequencies $f_{1}, f_{2}$, and $f_{3}$ of the peaks are read from Fig. $11 \mathrm{~b}$ as

$$
\begin{gathered}
f_{1}=19.53 \mathrm{~Hz} \\
f_{2}=2 \times 19.53 \mathrm{~Hz}=39.06 \mathrm{~Hz} \\
f_{3}=3 \times 19.53 \mathrm{~Hz}=58.59 \mathrm{~Hz}
\end{gathered}
$$

The peak at $50 \mathrm{~Hz}$ both before and after surge is associated with a grounding problem. The surge frequency is $19.53 \mathrm{~Hz}$, and the multiples of this frequency are believed to be higher-order harmonics of the nonlinear surge oscillation. As can be seen, the simulation was very accurate up to the third harmonic, also with respect to amplitude. The simulation also predicted the frequencies of the higher harmonics. This good match between experiment and simulation lead us to believe that the compressor characteristic was accurately computed, not only in the stable operating area, where comparison with experiment was possible, but also in the unstable part of the compressor map where no other experimental data are available.

The rotational speed of the compressor was also affected by the surge oscillations, but the oscillation of this signal was not visible from Fig. 11 due to noise. The power spectrum of the speed is shown in Fig. 12. In Fig. 12b, it can be seen that the speed signal had a strong component at $19.53 \mathrm{~Hz}$, the surge frequency. This component was not present before the surge event, as can be seen in Fig. 12a. Because the speed measurement included a relatively high level of noise, and the surge-induced speed oscillation was of a low amplitude due to the small plenum volume, it would be interesting to run this experiment with a larger volume to investigate the matching of the higher harmonics. Simulations show that by an increase of 
the plenum volume by a factor of 10 , the amplitude of the speed oscillation increases to $280 \mathrm{rpm}$.

\section{Conclusions}

A dynamic model of a variable speed compression system was derived and was validated by comparison with independent experimental data. The model is based on the underlying physics of the system and requires only a few parameters to be estimated from experimental data. A good fit was obtained by the use of a somewhat modified incidence loss term that is also closer to the underlying physics. To match the surge frequency, the duct length in the model was tuned. The unmodeled dynamics between the fuel flow signal and the drive torque was approximated with a first-order linear model. The compression system model includes a speed-dependent compressor characteristic. The derivation of this characteristic was done when the energy transfer was studied and when losses were taken into account. The calculated steady-state characteristic matched the measured one with very good accuracy, except near the choke line. To validate the dynamics of the model, two types of experiments were used: set-point changes induced by changes in the fuel flow or by adaption of the blowoff valve setting downstream of the compressor and compressor surge measurements. Both types of experiments verified the quality of the model. Transients were practically identical, and surge power spectral frequencies/amplitudes were predicted up to the third harmonic. The frequency and amplitude of the first harmonic of the surge-induced oscillation in rotational speed were also found.

The good agreement of the experimental results lead us to believe that the various losses for this compressor have been accurately modeled. Further work includes use of the model in active surge control by the use of energy-based methods.

\section{Acknowledgments}

J. T. Gravdahl acknowledges the financial support of ABB Industri AS of Norway. The authors gratefully acknowledge the invaluable help, advice, and suggestions of Corine Meuleman, Rick de Lange, and Harm van Essen during the experiments at Eindhoven University of Technology.

\section{References}

${ }^{1}$ Epstein, A., Williams, J. F., and Greitzer, E., "Active Suppression of Aerodynamic Instabilities in Turbomachines," Journal of Propulsion and Power, Vol. 5, No. 2, 1989, pp. 204-211.

${ }^{2}$ Willems, F., and Jager, B. D., "Modeling and Control of Compressor Flow Instabilities," IEEE Control Systems, Vol. 19, No. 5, 1999, pp. 8-18.

${ }^{3}$ Gravdahl, J., and Egeland, O., Compressor Surge and Rotating Stall: Modeling and Control, Advances in Industrial Control, Springer-Verlag, London, 1999.

${ }^{4}$ Paduano, J., Greitzer, E., and Epstein, A., "Compression System Stability and Active Control," Annual Reviews in Fluid Mechanics, Vol. 33, 2001, pp. 491-517.

${ }^{5}$ Greitzer, E., "Surge and Rotatin Stall in Axial Flow Compressors, Part I: Theoretical Compression System Model," Journal of Engineering for Power, Vol. 98, No. 2, 1976, pp. 190-198.

${ }^{6}$ Hansen, K., Jørgensen, P., and Larsen, P., "Experimental and Theoretical Study of Surge in a Small Centrifugal Compressor," Journal of Fluids Engineering, Vol. 103, No. 3, 1981, pp. 391-394.
${ }^{7}$ Fink, D., Cumpsty, N., and Greitzer, E., "Surge Dynamics in a Free-Spool Centrifugal Compressor System," Journal of Turbomachinery, Vol. 114, No. 2, 1992, pp. 321-332.

${ }^{8}$ Gravdahl, J., and Egeland, O., "Centrifugal Compressor Surge and Speed Control," IEEE Transactions on Control Systems Technology, Vol. 7, No. 5, 1999.

${ }^{9}$ Lozano, R., Borgliato, B., Egeland, O., and Maschke, B., Dissipative Systems Analysis and Control, Springer, London, 2000.

${ }^{10}$ Gravdahl, J., Egeland, O., and Vatland, S., "Drive Torque Actuation in Active Control of Centrifugal Compressors," Automatica, Vol. 38, No. 11, 2002, pp. 1881-1893.

${ }^{11}$ Gravdahl, J., Willems, F., Jager, B. D., and Egeland, O., "Modeling for Surge Control of Centrifugal Compressors: Comparison with Experiment," Proceedings of the 39th IEEE Conference on Decision and Control, IEEE Publications, Piscataway, NJ, 2000, pp. 1341-1346.

${ }^{12}$ van Essen, H., "Design of a Laboratory Gas Turbine Installation," Tech. Rept. WOC-WET 95.012, Eindhoven, Univ. of Technology, Eindhoven, The Netherlands, March 1995.

${ }^{13}$ van Essen, H., "Modelling and Model Based Control of Turbomachinery," Ph.D. Dissertation, Faculty of Mechanical Engineering, Eindhoven Univ. of Technology, Eindhoven, The Netherlands, Nov. 1998.

${ }^{14}$ Willems, F., "Modeling and Bounded Feedback Stabilization of Centrifugal Compressor Surge," Ph.D. Dissertation, Faculty of Mechanical Engineering, Eindhoven Univ. of Technology, Eindhoven, The Netherlands, June 2000 .

${ }^{15}$ Watson, N., and Janota, M., Turbocharging the Internal Combustion Engine, MacMillan, NewYork, 1982.

${ }^{16}$ Ferguson, T., The Centrifugal Compressor Stage, Butterworths, London, 1963.

${ }^{17}$ Futral, S., and Wasserbauer, C., "Off Design Performance Prediction with Experimental Verification for a Radial-Inflow Turbine," Tech. Rept. TN D-2621, NASA, Feb. 1965.

${ }^{18}$ Whitfield, A., and Wallace, F., "Study of Incidence Loss Models in Radial and Mixed-Flow Turbomachinery," Proceedings of the Conference on Heat and Fluid Flow in Steam and Gas Turbine Plant, Inst. of Mechanical Engineers, 1973, pp. 122-128.

${ }^{19}$ White, F., Fluid Mechanics, 2nd, ed., McGraw-Hill, New York, 1986.

${ }^{20}$ Strub, R., Bonciani, L., Borer, C., Casey, M., Cole, S., Cook, B., Kotzur, J., Simon, H., and Strite, M., "Influence of the Reynolds Number on the Performance of Centrifugal Compressors," Jornal of Turbomachinery, Vol. 109, No. 4, 1987, pp. 541-444.

${ }^{21}$ Johnston, J., and Dean, R., "Losses in Vaneless Diffusers of Centrifugal Compressors and Pumps. Analysis, Experiment and Design," Journal of Engineering for Power, Vol. 88, No. 1, Jan. 1966, pp. 49-62.

${ }^{22} \mathrm{Oh}$, H., Yoon, E., and Chung, M., "An Optimum Set of Loss Models for Performance Prediction of Centrifugal Compressors," Proceedings of the Institution of Mechanical Engineers, Part A: Journal of Power and Energy, Vol. 211, 1997, pp. 331-338.

${ }^{23}$ Thanapandi, P., and Prasad, R., "Performance Prediction and Loss Analysis of Low Specific Speed Submersible Pumps," Proceedings of the Institution of Mechanical Engineers: Part A: Journal of Power an Energy, Vol. 204, 1990, pp. 243-252.

${ }^{24}$ Day, I., "Axial Compressor Performance During Surge," Journal of propulsion and power, Vol. 10, No. 3, 1994, pp. 329-336.

${ }^{25}$ Badmus, O., Chowdhury, S., and Nett, C., "Nonlinear Control of Surgem Axial Compression Systems," Automatica, Vol. 32, No. 1, 1996, pp. 59-70.

${ }^{26}$ Cumpsty, N., Compressor Aerodynamics, Addition Wesley, Longman, Reading, MA, 1989.

${ }^{27}$ Mitra, S., Digital Signal Processing: A Computer-Based Approach, McGraw-Hill, New York, 1998. 